\title{
Importância da laserterapia no tratamento de feridas
}

\author{
Importance of lasertherapy in the treatment of wounds \\ Importancia de la láserapia en el tratamiento de heridas
}

Taiane Lima dos Santos ${ }^{1 *}$, Bruna Carla Pinheiro Ferreira Costa ${ }^{2}$, Cleuson Vieira Costa ${ }^{3}$, Edilene Bispo Gomes ${ }^{4}$, Laynara Suellem dos Santos Ripardo, ${ }^{5}$, Odileia Borges Quaresma ${ }^{6}$, Odemar Raimundo Gonçalves Baena Junior ${ }^{7}$, Silvio Douglas Medeiros Costa ${ }^{2}$, Simone Ribeiro Vieira ${ }^{8}$, Silvia Maria dos Santos Sousa².

\section{RESUMO}

Objetivo: Buscar na literatura científica evidências quanto aos benefícios da laserterapia no tratamento de feridas. Métodos: Trata-se de uma revisão integrativa da literatura. A busca dos estudos foi realizada nas bases de dados Literatura Latino Americana e do Caribe em Ciências da Saúde (LILACS) e Scientific Electronic Library Online (SCIELO), acessados através da Biblioteca Virtual em Saúde (BVS). A mostra final foi composta por 12 artigo condizentes com a questão desta pesquisa. Resultados: Pode-se observar que a maioria dos estudos incluídos nesta revisão teve uma abordagem quantitativo (9/75\%); e houve prevalência de revisão sistemática $(9 / 75 \%)$. Todos os artigos estavam no idioma português $(12 / 100 \%)$ e foram realizados no Brasil (12/100\%). Houve prevalência dos estudos publicados no ano 2021 (6/50\%). A análise dos estudos possibilitou evidenciar que o processo de bioestimulação provocada pela laserterapia ocorrem em nível molecular promovendo os principais efeitos fisiológicos que favorece a cicatrização da ferida, tais como a ação anti-inflamatória, neoangiogênese, proliferação epitelial e de fibroblastos, síntese e deposição de colágeno, revascularização e contração da ferida. Considerações finais: A laserterapia é um forte aliado no tratamento de feridas, pois este por meio de um processo celular acelera a cicatrização das feridas.

Palavras-chave: Cicatrização, Feridas, Lasers, Tratamento.

\begin{abstract}
Objective: Search the scientific literature for evidence on the benefits of laser therapy in the treatment of wounds. Methods: This is an integrative literature review. The search for studies was performed in the Latin American and Caribbean Literature in Health Sciences (LILACS) and Scientific Electronic Library Online (SCIELO) databases, accessed through the Virtual Health Library (VHL). The final sample consisted of 12 articles consistent with the question of this research. Results: It can be seen that most of the studies included in this review had a quantitative approach (9/75\%); and there was a prevalence of systematic review $(9 / 75 \%)$. All articles were in Portuguese (12/100\%) and were carried out in Brazil (12/100\%). There was a prevalence of studies published in $2021(6 / 50 \%)$. The analysis of the studies made it possible to evidence that the biostimulation process caused by laser therapy occurs at the molecular level, promoting the main physiological effects that favor wound healing, such as anti-inflammatory action, neoangiogenesis, epithelial and fibroblast proliferation, synthesis and deposition of collagen, revascularization and wound contraction. Final considerations: Laser therapy is a strong ally in wound treatment, as this through a cellular process accelerates wound healing.
\end{abstract}

Key words: Healing, Wounds, Lasers, Treatment.

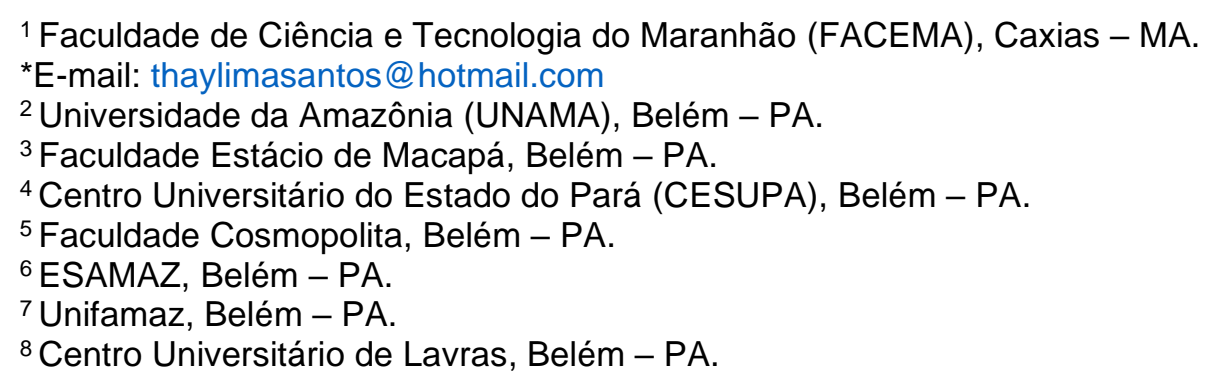




\section{RESUMEN}

Objetivo: Buscar en la literatura científica evidencia sobre los beneficios de la terapia con láser en el tratamiento de heridas. Métodos: Esta es una revisión integradora de la literatura. La búsqueda de estudios se realizó en las bases de datos de Literatura Latinoamericana y del Caribe en Ciencias de la Salud (LILACS) y Biblioteca Electrónica Científica en Línea (SCIELO), a las que se accede a través de la Biblioteca Virtual en Salud (BVS). La muestra final estuvo conformada por 12 artículos consistentes con la pregunta de esta investigación. Resultados: Se puede observar que la mayoría de los estudios incluidos en esta revisión tuvieron un enfoque cuantitativo $(9 / 75 \%)$; y hubo una prevalencia de revisión sistemática $(9 / 75 \%)$. Todos los artículos estaban en portugués (12/100\%) y se realizaron en Brasil (12/100\%). Hubo una prevalencia de estudios publicados en 2021 (6/50\%). El análisis de los estudios permitió evidenciar que el proceso de bioestimulación provocado por la terapia láser ocurre a nivel molecular, promoviendo los principales efectos fisiológicos que favorecen la cicatrización de heridas, como la acción antiinflamatoria, neoangiogénesis, proliferación epitelial y fibroblástica, síntesis y deposición de colágeno, revascularización y contracción de la

herida. Consideraciones finales: La terapia con láser es un fuerte aliado en el tratamiento de heridas, ya que esta a través de un proceso celular acelera la cicatrización de las heridas.

Palabras clave: Curación, Heridas, Láseres, Tratamiento.

\section{INTRODUÇÃO}

A cicatrização de feridas é um processo complexo e dinâmico que provoca alterações celulares e vasculares, resultando em um ciclo contínuo e prolongado de tratamento para propagação celular, formação e deposição de colágeno, síntese de elastina e revascularização, até a cicatrização da ferida (LIMA ES, et al., 2021).

A laserterapia é um recurso terapêutico usado para o tratamento de feridas que promove efeitos bioquímicos, bioelétricos e bioenergéticos e tem sido utilizado com o objetivo de acelerar o processo de cicatrização (MESTRE T, et al., 2020). Na terapia com laser utiliza-se um raio de baixa potência, que possibilita a aplicação não térmica e seu feixe eletromagnético sobre alguma área do corpo para fins terapêuticos, a terapia vem sendo usada como auxiliar para tratamentos convencionais ou isolada para o tratamento de patologias (BASTOS LLS, et al., 2017).

Em nível celular, a bioestimulação provocada pelo laser aumenta a ação da desidrogenase succínica, altera os níveis de prostaglandina, eleva a síntese de adenosina trifosfato (ATP) e a formação de energia, previne a necrose celular e ativa a proliferação de fibroblastos e macrófagos (RODRIGUES MFB, et al., 2020). O laser age inicialmente na célula, aumentando o metabolismo e proporcionando o aumento de granulação nos tecidos, regenerando as fibras nervosas, provocando a formação de novos vasos sanguíneos e regeneração dos linfáticos (FERREIRA ACD, et al., 2021).

A terapia com laser aumenta o fluxo sanguíneo na fase inicial da cicatrização e ativa mediadores inflamatórios para a lesão na fase de coagulação além de estimular a produção de colágeno nos estágios finais da cicatrização (SILVA JRM, et a., 2021). Além de contribuir na recuperação do aspecto estético da pele, a restauração da função ou área anatômica perdida resultante de uma lesão profunda, e proporcionar o alívio da dor e controle da inflamação, uma vez que promove a proliferação de fibroblastos, síntese de colágeno e epitelização (DAMANTE CA, et al., 2018).

Existe inúmeros tipos de laser indicados para cicatrização, dente eles destacam-se o Arseneto de Gálio (AsGa) - 904,0 nm, pulsado, feixe de luz invisível, potência de pico 15 a $30 \mathrm{~mW}$ e o Hélio-Neônio (HeNe) $632,8 \mathrm{~nm}$, contínuo, o feixe visível e a Potência de Pico (PP) localizada entre 2 a $10 \mathrm{~mW}$ (BARBOSA AC, et al., 2017).

A diferença estre os tipos de laser encontra-se no comprimento de onda e para serem usados devem se adequar ao local do ferimento para sua melhor performance. Desta forma deve ser levado em conta o aspecto e cor da pele do paciente e também deve depender do comprimento de onda, do nível de energia depositado, frequência de tratamento e estado do tecido irradiado (ANDRADE FSSD, et al., 2018). 
A terapia com laser é considerada uma terapia não invasiva, indolor e não térmica, a luz monocromática que é realizada pelo profissional enfermeiro como forma alternativa de tratamento de uma lesão. Segundo Brum MLB, et al. (2019), o cuidado de lesões é atribuição da enfermagem, sendo instituído pela Resolução do Conselho Federal de Enfermagem 501/2015.

Os cuidados a pacientes com feridas é uma especialidade da enfermagem, validada pela Sociedade Brasileira de Enfermagem Dermatológica (SOBEND) e Associação Brasileira de Estomaterapia (SOBEST), que tem o objetivo de capacitar os profissionais para tratar as diversas formas de lesões com conhecimentos específicos, técnicas, habilidades e abordagem holística (SILVA PC, et al., 2021).

De acordo com Ferreira AM, et al. (2020), para prestar um excelente cuidado a clientes portadores de feridas é necessária uma assistência interdisciplinar visto a diversidade de variáveis que envolve o cuidado de feridas. Tendo em visa que esta é uma atribuição desenvolvida pela enfermagem em sua prática diária, o fato torna estes profissionais mais indicados para prevenir, avaliar e tratar feridas.

Desta forma o enfermeiro é o profissional responsável por executar as etapas do cuidado em feridas, incluindo o acolhimento do paciente, a avaliação da ferida, bem como a escolha do tratamento a ser utilizado até a regressão da ferida, tendo este autônomia para executar todas as etapas com respaldo para tomada de decisões, sem a necessidade de auxilio de outro profissional de saúde (SILVEIRA PCL, et al., 2018).

No entanto é importante que o enfermeiro que se dedica a tratar feridas tenha conhecimento amplo dos materiais que serão utilizados no tratamento a sua disponibilidade no mercado assim como na fisiologia da cicatrização, compreendendo as etapas e ajustando o tratamento de acordo cada tipo de lesão (SILVA PC, et al. 2021).

O presente estudo teve o objetivo de buscar na literatura científica evidências quanto aos benefícios da laserterapia no tratamento de feridas.

\section{MÉTODOS}

O presente estudo trata-se de uma revisão integrativa da literatura, que buscou identificar os benefícios da laserterapia no tratamento de feridas. Esse tipo de estudo permite sintetizar resultados adquiridos em pesquisas sobre um tema ou questão, de forma sistemática, ordenada e abrangente.

Para a produção do estudo houve a definição do objetivo, o estabelecimento de critérios de inclusão e exclusão para a escolha da amostra, a determinação das informações a serem extraídas dos artigos selecionados, a análise e discussão dos resultados. Para orientar a elaboração do estudo formulou-se a seguinte questão: Quais evidências cientifica quanto os benefícios da laserterapia no tratamento de feridas?

A busca dos estudos utilizados na amostra foi realizada nas bases de dados Literatura Latino Americana e do Caribe em Ciências da Saúde (LILACS) e Scientific Electronic Library Online (SCIELO), acessados através da Biblioteca Virtual em Saúde (BVS). Os termos utilizados foram consultados nos Descritores em Ciência da Saúde (DeCS) e definidos como: Cicatrização, Feridas, Lasers, Tratamento.

Foram incluídos na busca nas bases de dados artigos disponíveis na íntegra, que foram publicados nos últimos 5 anos, no idioma que consentisse a temática do estudo. No entanto foi excluído da busca nas bases de dados os capítulos de livros, resumos, textos incompletos, teses e dissertações. A coleta dos dados ocorreu no mês de Agosto de 2021.

Os estudos foram pré-selecionados segundo os critérios de inclusão e exclusão no qual obteve-se 150 estudos como busca geral na base de dados LILACS, ao limitar a busca para texto completo e estudos que foram publicados nos últimos cinco anos obteve-se 31 estudos, destes foram analisados títulos e resultados onde apenas 9 estudos foi condizente com a questão desta pesquisa.

$\mathrm{Na}$ base SCIELO, foi encontrado 87 estudos como busca total, ao realizar a filtragem dos estudos que limita as pesquisas em texto completo e que foram publicados nos anos últimos cinco anos obteve-se 20 estudos, destes foram analisados títulos e resultado no qual apenas 3 estudos foi condizente com a questão 
desta pesquisa. Na fase final da busca dos estudos estes foram analisados quanto ao potencial de colaboração no estudo, avaliando o atendimento à questão norteadora da pesquisa, assim como os objetivos, amostra, método, desfechos, resultados e conclusão, resultando em 12 artigos.

\section{RESULTADOS E DISCUSSÃO}

$\mathrm{Na}$ elaboração do estudo foi incluído 14 artigos. Pode-se observar que a maioria dos estudos incluídos nesta revisão teve uma abordagem quantitativo $(9 / 75 \%)$ e houve prevalência de revisão sistemática $(9 / 75 \%)$. Todos os artigos estavam no idioma português (12/100\%) e foram realizados no Brasil (12/100\%). Houve prevalência dos estudos publicados no ano 2021 (6/50\%), como pode ser observado na Tabela 1.

Tabela 1 - Análise descritiva das produções científicas acerca dos benefícios da laserterapia no tratamento de feridas.

\begin{tabular}{l|c|c}
\hline \multicolumn{1}{c|}{ Variáveis } & N & $\%$ \\
\hline Abordagem do estudo & & 25 \\
\hline Qualitativo & 3 & 75 \\
Quantitativo & 9 & \\
\hline Delineamento da pesquisa & 3 & 25 \\
\hline Série de Casos & 9 & 75 \\
$\quad$ Revisão sistemática & 12 & 100 \\
\hline Idioma & & \\
\hline Português & 12 & 100 \\
\hline Procedência & 1 & \\
\hline Brasil & 1 & 8 \\
\hline Distribuição temporal & 4 & 8 \\
\hline 2018 & 6 & 33 \\
2019 & & 50 \\
2020 & & \\
2021 & & \\
\hline
\end{tabular}

Fonte: Santos TL, et al., 2021.

A Tabela 2 mostra que a fonte online LILACS com 09/75\% da amostra, foi a que mais disponibilizou estudos sobre a temática proposta em relação a outra fonte online SCIELO (03/25\%) foi a que menos disponibilizou estudos sobre o tema estudado.

Tabela 2 - Distribuição dos estudos incluídos na amostra referentes à fonte online de publicação.

\begin{tabular}{c|c|c}
\hline Fonte online & Número absoluto & $\%$ \\
\hline LILACS & 9 & 75 \\
SCIELO & 3 & 25 \\
\hline
\end{tabular}

Fonte: Santos TL, et al., 2021.

Pode-se observar uma grande variação no perfil da amostra dos artigos incluídos no estudo, no entanto em sua maioria estes buscaram avaliar os benefícios da laserterapia no tratamento de feridas, como pode ser observado no Quadro 1. 
Quadro 1 - Distribuição dos estudos incluídos segundo o autor, objetivos, resultados e conclusão.

\begin{tabular}{|c|c|c|c|}
\hline Autor & Objetivos & Resultados & Conclusão \\
\hline $\begin{array}{l}\text { Silva EM, et al. } \\
\qquad(2020) .\end{array}$ & $\begin{array}{l}\text { Descrever as vantagens e } \\
\text { desvantagens da aplicabilidade do } \\
\text { laser de baixa intensidade no reparo } \\
\text { tecidual. }\end{array}$ & $\begin{array}{l}\text { Dentre os lasers de baixa intensidade que são } \\
\text { utilizados nas intervenções, os mais citados foram } \\
\text { HeNe ( } n=2) \text {, AIGalnP ( } n=2) \text {, InGaAIP ( } n=2) \text { e HTM } \\
(n=2) \text {, sem superioridade de nenhum dos tipos. }\end{array}$ & $\begin{array}{l}\text { O uso do laser depende da educação em saúde } \\
\text { e outros fatores para obter resultados mais } \\
\text { satisfatórios. }\end{array}$ \\
\hline $\begin{array}{l}\text { Santos JMG, } \\
\text { et al. (2021). }\end{array}$ & $\begin{array}{l}\text { Descrever o uso do laser no } \\
\text { tratamento de lesão por pressão } \\
\text { mostrando seus benefícios, sua } \\
\text { eficácia e menos tempos no reparo } \\
\text { tecidual. }\end{array}$ & $\begin{array}{l}\text { Com relação a eficácia da ação do laser como } \\
\text { terapêutica principal na revitalização do tecido } \\
\text { lesionado, o sítio de aplicação do laser e lesões } \\
\text { apresentadas pelos artigos em maior prevalência na } \\
\text { região sacral. }\end{array}$ & $\begin{array}{l}\text { A laserterapia é um tratamento adjutório com } \\
\text { ações capaz de contribuir em resolutividade } \\
\text { acelerada, o seu uso vem trazendo um impacto } \\
\text { positivo, proporcionando bem-estar e qualidade } \\
\text { de vida aos } \\
\text { clientes. }\end{array}$ \\
\hline $\begin{array}{l}\text { Tallamini I, et } \\
\text { al. (2021). }\end{array}$ & $\begin{array}{l}\text { Investigar o uso da laserterapia como } \\
\text { tratamento complementar pela } \\
\text { enfermagem no tratamento de } \\
\text { feridas. }\end{array}$ & $\begin{array}{l}\text { A laserterapia é um tratamento adjutório com ações } \\
\text { capaz de contribuir em resolutividade acelerada, } \\
\text { decorrente do processo de cicatrização de lesões } \\
\text { tecidual, com evidencia na ação antiinflamatória, } \\
\text { analgésico e cicatrizantes, onde constitui uma atuação } \\
\text { dinâmica nos efeitos bioquímicos. }\end{array}$ & $\begin{array}{l}\text { É essencial o aprofundamento científico pela } \\
\text { enfermagem de pesquisas que empreguem a } \\
\text { intervenção pela laserterapia na cicatrização } \\
\text { tecidual. }\end{array}$ \\
\hline $\begin{array}{l}\text { Silva GM, et } \\
\text { al. (2021). }\end{array}$ & $\begin{array}{l}\text { Evidenciar o recurso terapêutico da } \\
\text { laserterapia para a eficácia do } \\
\text { tratamento das cicatrizes em } \\
\text { queimados. }\end{array}$ & $\begin{array}{l}\text { A aplicabilidade da técnica da laserterapia em cicatrizes } \\
\text { dos queimados garante vários benefícios no aspecto e } \\
\text { fatores fisiológicos das lesões, com maior evidencia no } \\
\text { seu reparo tecidual. }\end{array}$ & $\begin{array}{l}\text { As cicatrizes das queimaduras podem ser } \\
\text { tratadas com essa técnica provocando ações } \\
\text { anti-inflamatórias, analgésicas e melhora no } \\
\text { aspecto do tecido. }\end{array}$ \\
\hline
\end{tabular}




\begin{tabular}{|c|c|c|c|}
\hline Autor & Objetivos & Resultados & Conclusão \\
\hline $\begin{array}{l}\text { Lopes JW, et } \\
\text { al. (2020). }\end{array}$ & $\begin{array}{l}\text { Discorrer acerca dos efeitos da } \\
\text { laserterapia de baixa potência (LLLT) } \\
\text { direcionada para o tratamento do pé } \\
\text { diabético. }\end{array}$ & $\begin{array}{l}\text { A pesquisa sugere a irradiação de ondas no } \\
\text { comprimento de } 632.8 \mathrm{~nm} \text { a uma fluência de } 4 \mathrm{~J} / \mathrm{cm} 2 \\
\text { como parâmetro para o tratamento de feridas crônicas } \\
\text { possibilitando não só a redução do tempo de } \\
\text { cicatrização como também a manutenção da dor e da } \\
\text { perda de sensibilidade. }\end{array}$ & $\begin{array}{l}\text { O uso da laserterapia de baixa potência nos } \\
\text { pacientes diabéticos afligidos por úlceras nos } \\
\text { pés mostrou-se uma terapia promissora. }\end{array}$ \\
\hline $\begin{array}{l}\text { Oliveira DPT, } \\
\text { et al. }(2020) \text {. }\end{array}$ & $\begin{array}{l}\text { Realizar um levantamento de dados } \\
\text { dos principais artigos publicados com } \\
\text { intuito de adquirir conhecimento } \\
\text { sobre o uso do laser em traumas } \\
\text { mamilares. }\end{array}$ & $\begin{array}{l}\text { Conforme os artigos estudados pode-se observar que o } \\
\text { laser é um fator importante para a cicatrização. }\end{array}$ & $\begin{array}{l}\text { O laser é eficaz, porém mais estudos devem } \\
\text { ser realizados para ampliar o conhecimento. }\end{array}$ \\
\hline $\begin{array}{l}\text { Baracho CP, } \\
\text { et al. (2020). }\end{array}$ & $\begin{array}{l}\text { Avaliar a ação da utilização do laser } \\
\text { terapêutico na cicatrização de feridas } \\
\text { cutâneas. }\end{array}$ & $\begin{array}{l}\text { A pesquisa evidenciou que os comprimentos de onda } \\
\text { compreendidos entre } 632,8-1000 \mathrm{~nm} \text { apresentam } \\
\text { resultados mais satisfatórios no processo de reparação } \\
\text { tecidual. }\end{array}$ & $\begin{array}{l}\text { O laser tem ação satisfatória na cicatrização } \\
\text { das lesões cutâneas. }\end{array}$ \\
\hline $\begin{array}{l}\text { Monteiro DR, } \\
\text { et al. (2020). }\end{array}$ & $\begin{array}{l}\text { Analisar a efetividade da terapia a } \\
\text { laser de baixa potência no tratamento } \\
\text { de lesões por pressão em pacientes } \\
\text { adultos. }\end{array}$ & $\begin{array}{l}\text { O uso do laser mostrou-se uma modalidade promissora } \\
\text { no tratamento de lesões por pressão por proporcionar a } \\
\text { redução do tempo de cicatrização e melhora no aspecto } \\
\text { das feridas. No entanto, os estudos apresentam } \\
\text { distintos parâmetros de aplicação, comprimentos de } \\
\text { onda, densidade de energia e frequência de tratamento. }\end{array}$ & $\begin{array}{l}\text { Há a necessidade de novas pesquisas para } \\
\text { criar e elucidar padronizações ou protocolos, a } \\
\text { fim de otimizar a qualidade de atendimento aos } \\
\text { pacientes portadores de lesão por pressão. }\end{array}$ \\
\hline
\end{tabular}




\begin{tabular}{|c|c|c|c|}
\hline Autor & Objetivos & Resultados & Conclusão \\
\hline $\begin{array}{l}\text { Bavaresco T, } \\
\text { et al. (2019). }\end{array}$ & $\begin{array}{l}\text { Identificar a ação da terapia a laser de } \\
\text { baixa potência na cicatrização de } \\
\text { feridas. }\end{array}$ & $\begin{array}{l}\text { Verificou-se que a terapia a laser de baixa potência } \\
\text { propicia ações positivas na oxigenação, crescimento e } \\
\text { modulação celular devido à luz irradiada, que afeta os } \\
\text { processos metabólicos e produz bioestimulantes } \\
\text { celulares e vasculares essenciais ao processo de } \\
\text { reparo tecidual. }\end{array}$ & $\begin{array}{l}\text { A terapia a laser de baixa potência é um } \\
\text { tratamento adjuvante que acelera o processo } \\
\text { de reparação tecidual e promove benefícios ao } \\
\text { conforto dos pacientes. }\end{array}$ \\
\hline $\begin{array}{l}\text { Silva JRM, et } \\
\text { al. (2021). }\end{array}$ & $\begin{array}{l}\text { Comparar os efeitos dos diversos } \\
\text { protocolos que utilizam o Laser de } \\
\text { Baixa Potência na cicatrização de } \\
\text { feridas cutâneas. }\end{array}$ & $\begin{array}{l}\text { Foi possível verificar que o uso de protocolos é } \\
\text { fundamental para obter bons resultados, pois estes } \\
\text { mostram que existe diversas possibilidades de } \\
\text { tratamento. }\end{array}$ & $\begin{array}{l}\text { É necessário uma padronização na utilização } \\
\text { dos protocolos para ajudar na pratica clinica } \\
\text { dos profissionais da área. }\end{array}$ \\
\hline $\begin{array}{l}\text { Andrade } \\
\text { FSSD, et al. } \\
\text { (2018). }\end{array}$ & $\begin{array}{l}\text { Reunir e esclarecer quais os reais } \\
\text { efeitos da laserterapia de baixa } \\
\text { potência sobre feridas cutâneas. }\end{array}$ & $\begin{array}{l}\text { Doses compreendidas entre } 3-6 \mathrm{~J} / \mathrm{cm} 2 \text { e acima de } 10 \\
\mathrm{~J} / \mathrm{cm} 2 \text { foram mais eficazes. Os comprimentos de onda } \\
\text { compreendidos entre } 632,8-1000 \mathrm{~nm} \text { mostraram. } \\
\text { Resultados mais satisfatórios no processo de } \\
\text { cicatrização tecidual. }\end{array}$ & $\begin{array}{l}\text { O laser de baixa potência pode ser indicado } \\
\text { com segurança para acelerar a resolução de } \\
\text { feridas cutâneas, muito embora este fato esteja } \\
\text { intimamente ligado à eleição de parâmetros } \\
\text { como dose, tempo e comprimento de onda. }\end{array}$ \\
\hline $\begin{array}{l}\text { Ferreira ACD, } \\
\text { et al. (2021). }\end{array}$ & $\begin{array}{l}\text { Compreender o mecanismo de ação } \\
\text { da laserterapia na reparação tecidual } \\
\text { e, mais especificamente, na } \\
\text { angiogênese. }\end{array}$ & $\begin{array}{l}\text { A laserterapia atuando como fotobiomodulador, com } \\
\text { efeitos analgésicos, anti-inflamatórios, anti-edema e } \\
\text { antimicrobianos, e apresenta vantagens nas três etapas } \\
\text { do processo de cicatrização. }\end{array}$ & $\begin{array}{l}\text { A laserterapia oferece melhorias nos } \\
\text { mecanismos de resposta celular, no entanto é } \\
\text { necessária a realização de mais estudos } \\
\text { clínicos que demonstre o grande potencial } \\
\text { angiogênico da laserterapia em cada fase da } \\
\text { cicatrização. }\end{array}$ \\
\hline
\end{tabular}

Fonte: Santos TL, et al., 2021. 
A análise dos estudos possibilitou evidenciar os inúmeros benefícios da laserterapia no tratamento de feridas. Diante disso o estudo realizado por Silva EM, et al. (2020), ressalta as vantagem e desvantagem do uso do laser no tratamento de feridas. Segundo o autor a laserterapia é um método de terapia inovadora que acelera o tempo de fechamento das lesões e melhora o seu aspecto, reduz o desconforto, dor e exsudato, diminui significativamente o tamanho da lesão, a maceração e o eritema perilesão além de aumentar o tecido de epitelização e granulação.

De acordo com Ferreira ACD, et al. (2021), a laserterapia é uma alternativa terapêutica utilizada para reparação de tecidos lesionados, esta atua como fotobiomodulador, propicia os efeitos antimicrobianos, antiinflamatórios, anti-edema e analgésicos, favorece os mecanismos de resposta celular e proporciona benefícios nas três etapas do processo de cicatrização.

No entanto de acordo com o estudo de Silva EM, et al. (2020), para que a terapia com o laser seja eficácia é necessária a interferência de múltiplos fatores, dentre eles destaca-se os fatores de ordem econômica, social, suporte público, cultural, nutricional, técnica, individual e funcional, além disso pode ocorrer o aumento da área lesionada em casos de lesões contaminadas, fator este que favorece a dependência do autocuidado do paciente como um aliado ao tratamento.

Ao avaliar o efeito da laserterapiano tratamento de lesões por pressão o estudo realizado por Santos JMG, et al. (2021), evidenciou que o laser é um método eficaz que diminui o tempo de cicatrização e reduz as complicações das lesões por pressão. Segundo o estudo houve a cicatrização da ferida de $6 \mathrm{~cm}$ em até 5 semanas, além de proporcionar efeitos analgésicos e anti-inflamatório.

De acordo com Mestre T, et al., (2020), a laserterapia constitui uma atuação dinâmica que provoca efeito de proliferação celular e síntese de elementos constituintes da matriz extracelular, incluindo as ibrascolágenas, elásticas e reticulares, desta forma o aumento do metabolismo celular ocorre através da absorção molecular da luz do laser, designado a uma estimulação de síntese de colágeno, fotorreceptores mitocondriais e alterações dos níveis de ATP.

Desta forma a microcirculação sucede na pressão hidrostática capilar, com reabsorção do liquido extracelular e eliminação do acúmulo de metabólitos intermediários, função essa necessária para aumento do fluxo sanguíneo local, resultando em metabolitos fundamentais para regeneração (BARBOSA AC, et al. (2017).

Santos JMG, et al., (2021), ressalta em seu estudo que ao utilizar a terapêutica com laser é importante avaliar a maior abrangência de variáveis, para êxito no tratamento, incluindo as do próprio equipamento, pois no estudo quando realizado a comparação dos comprimentos de onda utilizados, verificou-se que o comprimento de onda entre $600 \mathrm{~nm}$ a $699 \mathrm{~nm}$ traz mais resultados positivos ao tratamento. Resultado similar foi evidenciado no estudo de Andrade FSSD, et al. (2018) no qual identificou em seu estudo que doses do laser entre $3-6 \mathrm{~J} / \mathrm{cm} 2$ foram mais eficazes e que doses acima de $10 \mathrm{~J} / \mathrm{cm} 2$ está associada a efeitos deletérios. No estudo os comprimentos de onda entre 632,8-1000nm também apresentam resultados mais satisfatórios no processo de cicatrização tecidual.

O fato corrobora com o estudo realizado por Tallamini L, et al. (2021), de acordo com a autora a laserterapia favorece a cicatrização das feridas por estimular a migração celular, atividade mitocondrial e proliferação fibroblástica, mantendo a viabilidade sem provocar prejuízos ou estresse celular, além de possuir ação antiinflamatória. No estudo a autora evidencio a eficácia do laser em feridas cirúrgicas, na qual pode-se observar a sua eficácia em aperfeiçoar o fechamento cirúrgico, reduzir infecção, dor e o período geral de cicatrização da ferida.

No que se refere a eficácia do laser quando utilizada no tratamento de queimaduras o estudo realizado por Silva GM, et al. (2021), evidenciou que a laserterapia promove benefícios na aparência da cicatriz, por seu efeitos anti-inflamatórios, por estimular o colágeno e promover função analgésica.

O autor afirma ainda que a terapia com o laser de baixa potência é um método que favorece o processo de reparo dos tecidos biológicos traumatizados, sendo este um processo de bioestimulação que ocorre a nível 
molecular promovendo os principais efeitos fisiológicos de anti-inflamatória, neoangiogênese, proliferação epitelial e de fibroblastos, síntese e deposição de colágeno, revascularização e contração da ferida (SILVA GM, et al., 2021).

Corrobora com o achado o estudo realizado por Lopes JW, et al. (2020), que observou melhora da perfusão tecidual local e do quadro álgico ao tratar lesões por pressão em pé diabético, além de maior estímulo a neovascularização e proliferação celular. No estudo ouve a comparação da eficácia do laser e do LED, no qual ao final de 10 sessões, observou-se que no grupo laser ouve a redução da ferida de $79.43 \%$ e com o uso do LED de $55.84 \%$, foi observado uma melhor cicatrização pelo laser.

Resultado similar foi observado por Monterio DR, et al. (2020), que também identificou melhor cicatrização da lesão por pressão com o uso do laser, de acordo com o autor a laserterapia traz bons resultados como terapia adjuvante, pois observou-se no estudo a redução da área lesionada ou sua total epitelização. No entanto o autor ressalta que tal resultado está relacionado a fatores como quantidade de sessões a que os pacientes foram expostos, quadro clínico, medidas corretas do aparelho, aderência ao tratamento, curativos e coberturas adequadas.

Corrobora com o achado a pesquisa de Oliveira DPT, et al. (2021), que buscou analisar o efeito da lasertarapia no tratamento de lesões mamilares, o estudo evidenciou que a terapia com laser de baixa potência é eficaz no cuidado de lesões mamilares como rachaduras, fissuras e escoriações, além de aliviar a dor durante a amamentação também ajuda na cicatrização.

O autor também ressalta que apesar da eficácia da laserterapia é importância que os profissionais tenham conhecimento quanto a utilização adequada do laser quanto à potência ( $\mathrm{W}$ ou $\mathrm{mW}$ ), modo (contínuo ou pulsado), pulso (frequência $\mathrm{Hz}$, duração do pulso nanossegundos), comprimento de onda ( $\lambda$ ), tipo de ponta e calibração do aparelho (OLIVEIRA DPT, et al., 2021),

Estudo realizado por Baracho CP, et al. (2020), evidenciou que doses compreendidas entre 3-6 J/cm2 apresentam ser mais eficazes, enquanto doses acima de $10 \mathrm{~J} / \mathrm{cm} 2$ está ligada a efeitos maléficos. Os comprimentos de onda compreendidos entre 632,8-1000nm mostraram resultados mais favoráveis na reparação tecidual. No estudo a laserterapia proporcionou a aceleração da cicatrização das feridas cutâneas, aumento do tecido epitelial, de granulação e uma diminuição de secreção e odor. Além de diminuir consideravelmente as dimensões da lesão durante o tratamento (BARACHO CP, et al., 2020).

De acordo com Bavaresco T, et al. (2019), os profissionais devem estar atentos quanto a dose em relação à densidade de energia $\left(\mathrm{J} / \mathrm{cm}^{2}\right)$ e de potência $\left(\mathrm{W} / \mathrm{cm}^{2}\right)$, área tratada ou área da ponta ativa $\left(\mathrm{cm}^{2}\right.$ ou $\left.\mathrm{mm}^{2}\right)$, tempo de aplicação (s), número de pontos tratados, número de joules por ponto e número total de joules por tratamento em protocolos para diferentes situações clínicas.

No que diz respeito aos protocolos Silva JRM, et al. (2021), ressalta em seu estudo a existência de diversos protocolos quanto a utilização do laser de baixa potência no tratamento de ferida, de acordo com o autor os protocolos apresentam bons resultados e diversas possibilidades de tratamento. No entanto o autor desta a necessidade de padronizar a utilização desse recurso com o intuito de facilitar a prática clinica dos profissionais da área, bem como melhorar a qualidade de vida dos indivíduos com lesões.

\section{CONSIDERAÇÕES FINAIS}

A análise dos estudos possibilitou identificar que a laserterapia é uma forte aliada no tratamento de feridas, pois esta por meio de um processo celular acelera a cicatrização das feridas. Pode-se evidenciar que a terapia com laser acelera o processo de cicatrização das feridas por provocar efeito anti-inflamatório, analgésico e regenerativos. A bioestimulação causada pelo laser aumentando o metabolismo, promovendo o aumento de granulação nos tecidos, regenerando as fibras nervosas, estimulando a formação de novos vasos sanguíneos e a regeneração dos linfáticos, favorecendo desta forma a cicatrização da ferida mais rapidamente. Diante disso o presente estudo é importante pois este buscou evidências cientificas que contribuam para 0 fortalecimento do conhecimento dos profissionais quanto ao uso do laserterapia no tratamento de feridas. 


\section{REFERÊNCIAS}

1. ANDRADE FSSD, et al. Efeitos da laserterapia de baixa potência na cicatrização de feridas cutâneas. Rev. Col. Bras. Cir., 2018; 41 (2): 129-133.

2. BARBOSA AC, et al. Laserterapia de baixa potência no tratamento de úlceras diabéticas. Acta Med Port, 2017; 24(5): 875-880.

3. BASTOS LLS, et al. Efeito da laserterapia na cicatrização de úlcera sacral dedecúbito. Biol. e saúde, campos dos Goytacazes, 2017; 18(5):56-57.

4. BARACHO CP, et al. Utilização do laser terapêutico na cicatrização de feridas cutâneas: uma revisão integrativa. Rev. Mult. Psic., 2020;154(53):732-738.

5. BAVARESCO T, et al. Terapia a laser de baixa potência na cicatrização de feridas. Rev. enferm. UFPE, 2019; 13(1):216-26.

6. BRUM MLB, et al. Protocolo de assistência de enfermagem a pessoas com feridas como instrumento para autonomia profissional. Rev Enferm UFSM, 2019; 5(1):50-57.

7. DAMANTE CA, et al. Terapia com laser em baixa intensidade na cicatrização de feridas - revisão de literatura. RFO, 2018; 13(3):88-93.

8. FERREIRA AC, et al. O enfermeiro e o tratamento de feridas: em busca da autonomiado cuidado. Arq Ciênc Saúde, 2020;15(3):105-9.

9. FERREIRA ACD, et al. A atuação da laserterapia na angiogênese e no reparo tecidual. Research, Society and Development, 2021;10(3): 346-350.

10. LIMA ES, et al. A eficácia da laserterapia no tratamento das radiodermatites: revisão integrativa. Research, Society and Development, 2021; 10(2):178-181.

11. LOPES JW, et al. Laserterapia de baixa potência no tratamento da úlcera do pé diabético. Revista UNILUS Ensino e Pesquisa, 2020; 17(49):34-38.

12. MESTRE T, et al. Cicatrização de feridas crónicas - algumas opções terapêuticas. Revista SPDV, 2020; 70(4): 890895.

13. MONTEIRO DR, et al. Efeito da terapia a laser no tratamento de lesões por pressão em pacientes adultos: revisão integrativa. Research, Society andDevelopment, 2020; 9(10):658-666.

14. OLIVEIRA DPT, et al. Um Estudo sobre o uso da Laserterapia em traumas mamilares. Humanidades \& Tecnologia (FINOM), 2021; 30(4):1809-1628.

15. RODRIGUES MFB, et al. Cicatrização de ferida cirúrgica tratada com laser de baixa intensidade: relato de caso. Arch Health Invest, 2020; 9(1):41-43.

16. SANTOS TL, et al. Terapia por pressão negativa no tratamento de feridas. Revista Eletrônica Acervo Saúde (REAS), 2019; 31(12):1-8.

17. SILVA JRM, et al. Análise comparativa dos efeitos do laser de baixa potência na cicatrização de lesões cutâneas: revisão sistemática. Brazilian Journal of Health Review, 2021; 4(3):13949-13960.

18. SILVA PC, et al. A atuação do enfermeiro no tratamento de feridas. Brazilian Journal of Health Review, 2021; 4(2): 4815-4822.

19. SILVA EM, et al. Vantagens e desvantagens da aplicabilidade do laser de baixa intensidade no reparo tecidual. Pesquisa em Saúde e Enfermagem: Inovação à ciência, 2020; 56(8):45-67.

20. SANTOS JMG, et al. O laser no tratamento de lesão por pressão. Research, Society and Development, 2021; 10(9):291-299.

21. SILVA GM, et al. Laserterapia no tratamento de cicatrizes em pacientes queimados. Revista Liberum Accessum, 2021; 9(2):40-50.

22. SILVEIRA PCL, et al. Efeitos da laserterapia de baixa potência na resposta oxidativa epidérmica induzida pela cicatrização de feridas. Rev Bras Fisioter, 2018; 13(4):281-7.

23. TALLAMINI I, et al. Processo de cicatrização e efeito da laserterapia de baixa potência: revisão integrativa. Passo Fundo, 2021;1(1):123-137. 